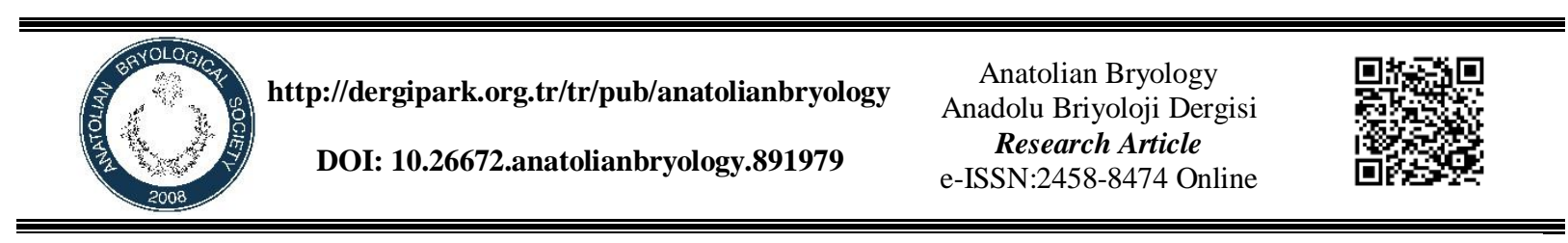

\title{
Determination of Heavy Metal Levels in Some Moss Samples Collected from Near the Highways in Burdur Province
}

\author{
Nevzat BATAN $^{1 *}$ (D), Turan ÖZDEMIR ${ }^{2}$ (D), Ekrem SARALIOĞLU ${ }^{3}$ iD, Nilay AKÇAY ${ }^{4}$ iD, Durali \\ MENDIL $^{5}$ iD \\ ${ }^{1}$ Karadeniz Technical University, Maçka Vocational School, Trabzon, TURKEY \\ ${ }^{2}$ Karadeniz Technical University, Biology Department, Faculty of Science, Trabzon, TURKEY \\ ${ }^{3}$ Artvin Coruh University, Department of Geomatics Engineering, Division of Remote Sensing, Artvin, TURKEY \\ ${ }^{4}$ Recep Tayyip Erdoğan University, Department of Physics, Faculty of Art and Science, Rize, TURKEY \\ ${ }^{5}$ Gaziosmanpasa University, Chemistry Department, Faculty of Science and Art, Tokat, TURKEY
}

\begin{abstract}
Received: 8 March $2021 \quad$ Revised: 23 March 2021 $\quad$ Accepted: 24 March 2021
Abstract

The main sources of atmospheric heavy metals deposition are agricultural activities, fossil fuels, central heating stations and the exhaust gases of motor vehicles. In this study, the total of 29 mosses samples were collected from contaminated points near the Isparta-Antalya, Burdur-Denizli, Burdur-Afyonkarahisar, Burdur-Fethiye (Muğla) and Burdur-Antalya highways (2-20m) and uncontaminated areas in 2012. Samples were analyzed by flame and graphite furnace atomic absorption spectrometry (AAS) later microwave digestion methods. The highest accumulation levels detected as $\mu \mathrm{g} . \mathrm{g}^{-1} \mathrm{Fe}(1419.02)>\mathrm{Zn}(158.44)>\mathrm{Mn}(362.77)>\mathrm{Ni}(109.53)>\mathrm{Cr}(60.51)>\mathrm{Pb}$ (30.38) $>\mathrm{Cd}(35.866)>\mathrm{Cu}$ (25.17) for mosses. Element levels are differ depending on moss species and the location of collecting localities.
\end{abstract}

Keywords: Biomonitoring, Heavy metals, Contamination, Mosses

\section{Burdur ilinde Karayollarının Yakınından Toplanan Bazı Karayosunu Örneklerinde Ağır Metal Seviyelerinin Belirlenmesi}

$\ddot{\mathbf{O z}}$

Atmosferik Ağır metal birikiminin ana kaynakları, tarımsal faaliyetler, fosil yakıtlar, merkezi ısıtma istasyonları ve bölgedeki motorlu taşıtların egzoz gazlarıdır. Bu çalışmada, 2012 yılında Isparta-Antalya, Burdur-Denizli, Burdur-Afyonkarahisar, Burdur-Fethiye (Muğla) ve Burdur-Antalya karayollarının yakınından (2-20m) kirlenmiş bölgeden ve bu yollara uzak noktalardan temiz bölgeden 29 karayosunu örneği toplanmıştır. Örnekler mikrodalga çözünürleştirme sonrasında alev ve grafit firın atomik absorpsiyon spektrometresi (AAS) metodu ile analiz edilmiştir. Karayosunları için en yüksek birikim seviyeleri $\mu \mathrm{g} . \mathrm{g}^{-1}$ olarak şu şekilde tespit edildi; Fe $(1419.02)>\mathrm{Mn}$ $(362.77)>\mathrm{Zn} \quad(158.44)>\mathrm{Ni} \quad(109.53)>\mathrm{Cr} \quad(60.51)>\mathrm{Pb} \quad(30.38)>\mathrm{Cd}(35.866)>\mathrm{Cu} \quad(25.17)$. Element seviyesi, karayosunu türlerine ve toplanma lokalitelerinin durumuna bağlı olarak farklıdır.

Anahtar kelimeler: Biyolojik izleme, Ağır metaller, Kontaminasyon, Karayosunu

\footnotetext{
* Corresponding author: nevzatbatan@gmail.com

(C) 2021 All rights reserved / Tüm haklarl saklıdır.

To cite this article: Batan N. Özdemir T. Saralıoğlı S. Akçay N. Mendil D. 2021. Determination of Heavy Metal Levels in Some Moss Samples Collected from Near the Highways in Burdur Province. Anatolian Bryology. 7:1, 33-43.

(c) (1) () This work is licensed under a Creative Commons Attribution Non-Commercial 4.0 International License.
} 


\section{Introduction}

Air pollution reaching large size in every day is a significant problem that concerns all countries, developed and developing. The major sources of air pollution are traffic (especially diesel vehicles), industrial sectors (from brick making to oil and gas production), power plants, cooking or heating with soil fuels (e.g. coal, wood, crop waste), forest fires and burning of municipal waste and agricultural residues (Akçay et al., 2016). Air pollutants resulting from natural and some human activities reach the atmosphere, and while they are carried as cloud or smoke bundle at first, they also adversely affect the living life with dilution and precipitation (Akçay et al., 2016). Many researches are reported that air pollution caused respiratory diseases, acute irritation of the eyes, cardiovascular disorders, abnormalities in the nervous system, development of cancer, and deaths (Akçay et al., 2016). It is also known that air pollution threatens the plant and animal life, and led to contamination of water (Akçay et al., 2016).

All organisms need different amounts of heavy metals. Humans need iron, cobalt, copper, manganese, molybdenum, and zinc at all times (Farid et al., 2015). The whole metals can be toxic at higher concentrations (Farid et al., 2015). Extreme levels can be damaging to every organism. Some of them, like mercury, plutonium, and lead are toxic metals, which have no known vital or beneficial effect on organisms, and their accumulation with time in the bodies of animals can reason serious diseases (Farid et al., 2015).

Some elements are necessary for anatomical and physiological functions for the human body, but some of the others such as cadmium and lead are nonessential metals, so little levels can be toxic.

Iron has many properties that make it a suitable structure for the oxygen-carrying protein, hemoglobin, in the red blood cells.
Sodium, potassium, magnesium, phosphorus, calcium, and many other elements constitute substantial elements of the body. Calcium and phosphorus are important component of the skeleton, teeth and bones. Calcium in minute concentrations provides common blood coagulation. Magnesium is an essential element to stimulate numerous enzyme activities, regulates contraction and sends impulses by nerve cells (Strain and Cashman, 2009).

Mosses are bio-monitors suitable for air pollutants because of their anatomical structures (Onianwa, 2001; Zeichmeister et al., 2003; Chakrabortty and Paratkar, 2006). They haven't got real roots and thus, they can't benefit use the soil to get their nutrient. Mosses absorb minerals from the atmosphere by all surface. Minerals uptake of mosses from the atmosphere is easier by their poorly developed cuticle. Their high absorbency feature improves adsorption. Undeveloped transmission bundles let better adsorption than vascular plants (Chakrabortty and Paratkar, 2006). The adhering of the particles depend on the size of the particles and the surface structure of the mosses (Chakrabortty and Paratkar, 2006; Brown and Bates, 1990; Tyler, 1990).

Burdur is located in the Mediterranean region of Turkey (Figure 1), and it has main roads connectig Mediterranean, Aegean and Central Anatolia regions. Up to date, there is no data about the level of heavy metal pollution from Burdur and its environment. So, in this study, the levels of heavy metals $(\mathrm{Cu}, \mathrm{Pb}, \mathrm{Cd}$, $\mathrm{Zn}, \mathrm{Fe}, \mathrm{Cr}, \mathrm{Ni}$, and $\mathrm{Mn}$ ) in some moss samples collected at the polluted (near the roadside Burdur-Denizli, Burdur-Muğla and Burdur-Antalya) and unpolluted locations around Burdur, by using flame and graphite furnace atomic absorption spectrometry (AAS) after microwave digestion. 


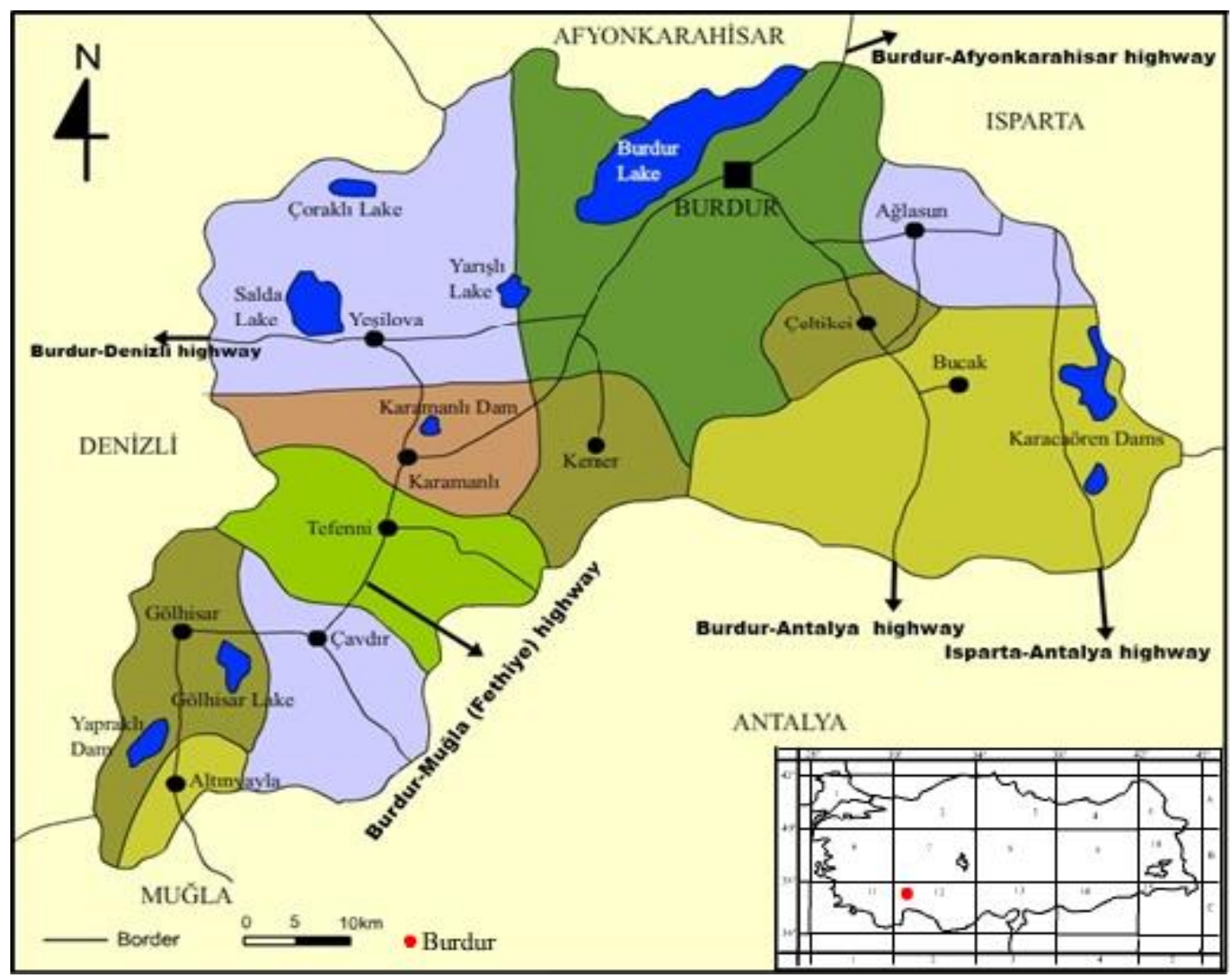

Figure 1. Geographical location of the Burdur

\section{Materials and Methods}

2.1. Sampling: Moss specimens were collected at the unpolluted ( far $50 \mathrm{~km}$ from city centre ) area and near $(2-20 \mathrm{~m})$ from high-density traffic roads and the city centre of Burdur Province. Samples were stored in polyethylene bottles until they analyzed.

2.2. Analytical procedure for AAS: The moss samples were dried at $105{ }^{\circ} \mathrm{C}$ for $24 \mathrm{~h}$ at oven. Dried samples were homogenized (Homogenization was carried out by grinding plants to a powder, using an acid-washed mortar and pestle). Milestone Ethos D microwave closed system was used in this study. 0.25 $\mathrm{g}$ of sample was digested with $6 \mathrm{~mL}$ of $\mathrm{HNO}_{3}(65 \%)$, $2 \mathrm{~mL}$ of $\mathrm{H}_{2} \mathrm{O}_{2}(30 \%)$ and $1 \mathrm{~mL} \mathrm{HF} \mathrm{(39 \% )} \mathrm{in}$ microwave digestion system for $27 \mathrm{~min}$ and diluted to $25 \mathrm{~mL}$ with deionizer water (digestion steps for microwave system were applied as $6 \mathrm{~min}$ for $250 \mathrm{~W}, 6$ min for $400 \mathrm{~W}, 6 \mathrm{~min}$ for $650 \mathrm{~W}, 6 \mathrm{~min}$ for $250 \mathrm{~W}$, vent: 3 min, respectively). A blank digest was carried out in the same way. In this study Unicam Model AA-929 model atomic absorption spectrometer was used for the elemental analysis. $\mathrm{Cu}, \mathrm{Pb}, \mathrm{Cd}, \mathrm{Zn}, \mathrm{Fe}, \mathrm{Cr}, \mathrm{Ni}$ and $\mathrm{Mn}$ were analyzed with flame atomic absorption spectrometry (FAAS). The concentration of elements in samples was determined using single element hollow-cathode lamps and air/acetylene burner head. $\mathrm{N}_{2} \mathrm{O}$ /acetylene-burner was used for chromium.

Double-deionizer water was used for all dilutions. $\mathrm{HNO}_{3}, \mathrm{H}_{2} \mathrm{O}_{2}$, and $\mathrm{HF}$ were of suprapure quality (Merck).

\section{Result and Discussion}

All element concentrations were analyzed as a dry weight as $\mu \mathrm{g} . \mathrm{g}^{-1}$. The standard deviations were found less than $10 \%$ for each elements. T-test was used in this research $(\mathrm{p}<0.05)$. The concentrations of $\mathrm{Cu}, \mathrm{Pb}, \mathrm{Cd}$, $\mathrm{Zn}, \mathrm{Fe}, \mathrm{Cr}, \mathrm{Ni}$ and $\mathrm{Mn}$ in moss samples were given in Table 1. The order amount of elements in moss samples were detected as $\mathrm{Fe}>\mathrm{Mn}>\mathrm{Zn}>\mathrm{Ni}>\mathrm{Cr}>\mathrm{Cd}>\mathrm{Pb}>\mathrm{Cu}$ 
Table 1. Descriptions of the stations in Burdur (Turkey)

\begin{tabular}{|c|c|c|c|c|}
\hline $\begin{array}{l}\text { Sample } \\
\text { Number }\end{array}$ & Moss species & Station & Lokality & $\begin{array}{l}\text { Altitude } \\
\quad(\mathbf{m})\end{array}$ \\
\hline 1 & Homalothecium sericeum & Between Beşkonak and Kocaaliler & $\begin{array}{l}37^{\circ} 20^{\prime} 52.64^{\prime \prime K} / \\
30^{\circ} 44^{\prime} 12.45^{\prime \prime} \mathrm{D}\end{array}$ & 846 \\
\hline 2 & Homalothecium sericeum & Salda Lake(Burdur-Denizli highway) & $\begin{array}{l}37^{\circ} 30^{\prime} 38.51^{\prime \prime K} / \\
29^{\circ} 41^{\prime} 33.31^{\prime \prime D}\end{array}$ & 1180 \\
\hline 3 & Homalothecium sericeum & $\begin{array}{l}\text { Karacaören (Isparta-Antalya } \\
\text { highway) }\end{array}$ & $\begin{array}{l}37^{\circ} 23^{\prime} 17.60^{\prime \prime K} / \\
30^{\circ} 49^{\prime} 58.76^{\prime \prime} \mathrm{D}\end{array}$ & 455 \\
\hline 4 & Bryum capillare & Between Beşkonak and Kocaaliler & $\begin{array}{l}37^{\circ} 20^{\prime} 52.64^{\prime \prime} \mathrm{K} / \\
30^{\circ} 44^{\prime} 12.45^{\prime \prime} \mathrm{D}\end{array}$ & 846 \\
\hline 5 & Bryum capillare & $\begin{array}{l}\text { Exit of Burdur (Burdur- Muğla, } \\
\text { Fethiye highway) }\end{array}$ & $\begin{array}{l}37^{\circ} 41^{\prime} 48.10^{\prime \prime} \mathrm{K} / \\
30^{\circ} 12^{\prime} 15.77^{\prime \prime D}\end{array}$ & 874 \\
\hline 6 & Syntrichia ruralis & Between Beşkonak and Kocaaliler & $\begin{array}{l}37^{\circ} 20^{\prime} 52.64^{\prime \prime} \mathrm{K} / \\
30^{\circ} 44^{\prime} 12.45^{\prime \prime} \mathrm{D}\end{array}$ & 846 \\
\hline 7 & Syntrichia ruralis & Salda gölü (Burdur-Denizli highway) & $\begin{array}{l}37^{\circ} 30^{\prime} 38.51^{\prime \prime K} / \\
29^{\circ} 41^{\prime} 33.31^{\prime \prime} \mathrm{D}\end{array}$ & 1180 \\
\hline 8 & Syntrichia ruralis & $\begin{array}{l}\text { Exit of Bucak (Burdur-Antalya } \\
\text { highway) }\end{array}$ & $\begin{array}{l}37^{\circ} 26^{\prime} 39.33^{\prime \prime K} / \\
30^{\circ} 39^{\prime} 19.98^{\prime \prime} \mathrm{D}\end{array}$ & 874 \\
\hline 9 & Syntrichia ruralis & $\begin{array}{l}\text { Exit of Burdur(Burdur-Afyon } \\
\text { highway) }\end{array}$ & $\begin{array}{l}37^{\circ} 45^{\prime} 05.79^{\prime \prime} \mathrm{K} / \\
30^{\circ} 19^{\prime} 56.37^{\prime \prime} \mathrm{D}\end{array}$ & 950 \\
\hline 10 & Syntrichia ruralis & $\begin{array}{l}\text { Exit of Burdur(Burdur -Fethiye } \\
\text { highway) }\end{array}$ & $\begin{array}{l}37^{\circ} 41^{\prime} 48.10^{\prime \prime} \mathrm{K} / \\
30^{\circ} 12^{\prime} 15.77^{\prime \prime} \mathrm{D}\end{array}$ & 874 \\
\hline 11 & Syntrichia ruralis & $\begin{array}{l}\text { Gölhisar (Burdur-Muğla, Fethiye } \\
\text { highway) }\end{array}$ & $\begin{array}{l}37^{\circ} 08^{\prime} 59.17 " \mathrm{~K} / \\
29^{\circ} 36^{\prime} 4071^{\prime \prime D}\end{array}$ & 970 \\
\hline 12 & Syntrichia ruralis & $\begin{array}{l}\text { Karamanl1(Burdur-Muğla, Fethiye } \\
\text { highway) }\end{array}$ & $\begin{array}{l}37^{\circ} 21^{\prime} 01.19^{\prime \prime} \mathrm{K} / \\
29^{\circ} 48^{\prime} 43.97^{\prime \prime} \mathrm{D}\end{array}$ & 1130 \\
\hline 13 & Syntrichia ruralis & $\begin{array}{l}\text { Hacilar(Burdur-Muğla, Fethiye } \\
\text { highway) }\end{array}$ & $\begin{array}{l}37^{\circ} 34 ' 32.57^{\prime \prime} \mathrm{K} / \\
30^{\circ} 05^{\prime} 27.06^{\prime \prime} \mathrm{D}\end{array}$ & 955 \\
\hline 14 & Syntrichia ruralis & $\begin{array}{l}\text { Exit of Tefenni (Burdur-Muğla, } \\
\text { Fethiye highway) }\end{array}$ & $\begin{array}{l}37^{\circ} 18^{\prime} 56.25^{\prime \prime K} / \\
29^{\circ} 45^{\prime} 35.66^{\prime \prime} \mathrm{D} \\
\end{array}$ & 1210 \\
\hline 15 & Syntrichia ruralis & $\begin{array}{l}\text { Çavdır (Burdur-Muğla, Fethiye } \\
\text { highway) }\end{array}$ & $\begin{array}{l}37^{\circ} 09^{\prime} 44.61 " \mathrm{~K} / \\
29^{\circ} 40^{\prime} 07.88^{\prime \prime} \mathrm{D}\end{array}$ & 1065 \\
\hline 16 & Syntrichia ruralis & $\begin{array}{l}\text { Karacaören (Isparta-Antalya } \\
\text { highway) }\end{array}$ & $\begin{array}{l}37^{\circ} 23^{\prime} 17.60^{\prime \prime} \mathrm{K} / \\
30^{\circ} 49^{\prime} 58.76 " \mathrm{D}\end{array}$ & 455 \\
\hline 17 & Syntrichia ruralis & Ağlasun (Isparta-Antalya highway) & $\begin{array}{l}37^{\circ} 38^{\prime} 23.65^{\prime \prime} \mathrm{K} / \\
30^{\circ} 22^{\prime} 23.28^{\prime \prime} \mathrm{D}\end{array}$ & 1216 \\
\hline 18 & Grimmia orbicularis & Between Beşkonak and Kocaaliler & $\begin{array}{l}37^{\circ} 20^{\prime} 52.64^{\prime \prime} \mathrm{K} / \\
30^{\circ} 44^{\prime} 12.45^{\prime \prime} \mathrm{D}\end{array}$ & 846 \\
\hline 19 & Grimmia orbicularis & $\begin{array}{l}\text { Exit of Bucak (Burdur-Antalya } \\
\text { highway) }\end{array}$ & $\begin{array}{l}37^{\circ} 26^{\prime} 39.33^{\prime \prime K} / \\
30^{\circ} 39^{\prime} 19.98^{\prime \prime} \mathrm{D}\end{array}$ & 874 \\
\hline 20 & Grimmia orbicularis & $\begin{array}{l}\text { Exit of Burdur (Burdur-Afyon } \\
\text { highway) }\end{array}$ & $\begin{array}{l}37^{\circ} 45^{\prime} 05.79^{\prime \prime} \mathrm{K} / \\
30^{\circ} 19^{\prime} 56.37^{\prime \prime} \mathrm{D}\end{array}$ & 950 \\
\hline 21 & Grimmia orbicularis & $\begin{array}{l}\text { Hacılar(Burdur-Muğla, Fethiye } \\
\text { highway) }\end{array}$ & $\begin{array}{l}37^{\circ} 34^{\prime} 32.57^{\prime \prime} \mathrm{K} / \\
30^{\circ} 05^{\prime} 27.06^{\prime \prime} \mathrm{D}\end{array}$ & 955 \\
\hline 22 & Homalothecium lutescens & Between Beşkonak and Kocaaliler & $\begin{array}{l}37^{\circ} 20^{\prime} 52.64^{\prime \prime} \mathrm{K} / \\
30^{\circ} 44^{\prime} 12.45^{\prime \prime} \mathrm{D}\end{array}$ & 846 \\
\hline 23 & Homalothecium lutescens & $\begin{array}{l}\text { Exit of Bucak (Burdur-Antalya } \\
\text { highway) }\end{array}$ & $\begin{array}{l}37^{\circ} 26^{\prime} 39.33^{\prime \prime K} / \\
30^{\circ} 39^{\prime} 19.98^{\prime \prime} \mathrm{D}\end{array}$ & 874 \\
\hline 24 & Homalothecium lutescens & $\begin{array}{l}\text { Exit of Burdur (Burdur -Afyon } \\
\text { highway) }\end{array}$ & $\begin{array}{l}37^{\circ} 45^{\prime} 05.79^{\prime \prime} \mathrm{K} / \\
30^{\circ} 19^{\prime} 56.37^{\prime \prime} \mathrm{D}\end{array}$ & 950 \\
\hline 25 & Homalothecium lutescens & $\begin{array}{l}\text { Gölhisar(Burdur-Muğla, Fethiye } \\
\text { highway) }\end{array}$ & $\begin{array}{l}37^{\circ} 08^{\prime} 59.17^{\prime \prime K} / \\
29^{\circ} 36^{\prime} 4071^{\prime \prime} \mathrm{D}\end{array}$ & 970 \\
\hline 26 & Homalothecium lutescens & $\begin{array}{l}\text { Karamanl1(Burdur-Muğla, Fethiye } \\
\text { highway) }\end{array}$ & $\begin{array}{l}37^{\circ} 21^{\prime} 01.19^{\prime \prime K} / \\
29^{\circ} 48^{\prime} 43.97^{\prime \prime} \mathrm{D}\end{array}$ & 1130 \\
\hline 27 & Homalothecium lutescens & $\begin{array}{l}\text { Hacilar(Burdur-Muğla, Fethiye } \\
\text { highway) }\end{array}$ & $\begin{array}{l}37^{\circ} 34^{\prime} 32.57^{\prime \prime K} / \\
30^{\circ} 05^{\prime} 27.06^{\prime \prime} \mathrm{D}\end{array}$ & 955 \\
\hline 28 & Homalothecium lutescens & $\begin{array}{l}\text { Exit of Tefenni (Burdur-Muğla, } \\
\text { Fethiye highway) }\end{array}$ & $\begin{array}{l}37^{\circ} 18^{\prime} 56.25^{\prime \prime} \mathrm{K} / \\
29^{\circ} 45^{\prime} 35.66^{\prime \prime} \mathrm{D}\end{array}$ & 1210 \\
\hline 29 & Homalothecium lutescens & $\begin{array}{l}\text { Çavdır(Burdur-Muğla, Fethiye } \\
\text { highway) }\end{array}$ & $\begin{array}{l}37^{\circ} 09^{\prime} 44.61 " \mathrm{~K} / \\
29^{\circ} 40^{\prime} 07.88^{\prime \prime} \mathrm{D}\end{array}$ & 1065 \\
\hline
\end{tabular}


The higher element accumulations were found 1535.4 $\mu \mathrm{g} . \mathrm{g}^{-1}$ (Fe), $362.77 \mu \mathrm{g} \cdot \mathrm{g}^{-1}(\mathrm{Mn}), 158.44 \mu \mathrm{g} \cdot \mathrm{g}^{-1}(\mathrm{Zn})$, $109.53 \mu \mathrm{g} . \mathrm{g}^{-1}(\mathrm{Ni}), 60.51 \mu \mathrm{g} \cdot \mathrm{g}^{-1}(\mathrm{Cr}), 35.86 \mu \mathrm{g} \cdot \mathrm{g}^{-1}$ (Cd), $30.38 \mu \mathrm{g} \cdot \mathrm{g}^{-1}(\mathrm{~Pb})$, and $25.17 \mu \mathrm{g} \cdot \mathrm{g}^{-1}(\mathrm{Cu})$. The concentrations near the highway edge and farmland (polluted area) were higher than the control area (Unpolluted area). Accumulation of iron, manganese, zinc, copper, chromium, nickel, lead and cadmium are the highest on the traffic roadsides and farmland (Table 2 ). The concentration of trace metals in the samples are depended on the species of mosses and the location of the collected points. The highest element accumulation was found in Homalothecium sericeum for Fe (1535.4 $\left.\mu \mathrm{g} . \mathrm{g}^{-1}\right), \mathrm{Mn}\left(233.6 \mu \mathrm{g} . \mathrm{g}^{-1}\right), \mathrm{Zn}\left(158.44 \mu \mathrm{g} . \mathrm{g}^{-1}\right), \mathrm{Ni}(87$ $\left.\mu \mathrm{g} . \mathrm{g}^{-1}\right), \mathrm{Cr}\left(60.51 \mu \mathrm{g} . \mathrm{g}^{-1}\right), \mathrm{Cu}\left(24.02 \mu \mathrm{g} . \mathrm{g}^{-1}\right), \mathrm{Pb}(11.35$ $\left.\mu \mathrm{g} . \mathrm{g}^{-1}\right), \mathrm{Cd}\left(9.185 \mu \mathrm{g} . \mathrm{g}^{-1}\right)$, Homalothecium lutescens Fe $\left(1271.93 \mu \mathrm{g} . \mathrm{g}^{-1}\right), \mathrm{Mn}\left(362.77 \mu \mathrm{g} . \mathrm{g}^{-1}\right), \mathrm{Zn}$ ( 69.59 $\left.\mu \mathrm{g} . \mathrm{g}^{-1}\right)$, Ni $\left(109.53 \mu \mathrm{g} . \mathrm{g}^{-1}\right), \mathrm{Cr}\left(60.51 \mu \mathrm{g} . \mathrm{g}^{-1}\right), \mathrm{Cu}$ (18.73 $\left.\mu \mathrm{g} . \mathrm{g}^{-1}\right), \mathrm{Pb}\left(27.20 \mu \mathrm{g} . \mathrm{g}^{-1}\right), \mathrm{Cd}\left(35.866 \mu \mathrm{g} . \mathrm{g}^{-1}\right)$, Grimmia orbicularis for $\mathrm{Fe}\left(856.82 \mu \mathrm{g} . \mathrm{g}^{-1}\right), \mathrm{Mn}$ (213.17 $\left.\mu \mathrm{g} . \mathrm{g}^{-1}\right), \mathrm{Zn}\left(52.13 \mu \mathrm{g} . \mathrm{g}^{-1}\right)$, Ni (57.36 $\left.\mu \mathrm{g} . \mathrm{g}^{-1}\right)$,
$\mathrm{Cr}\left(34.85 \mu \mathrm{g} \cdot \mathrm{g}^{-1}\right), \mathrm{Cu}\left(20.64 \mu \mathrm{g} . \mathrm{g}^{-1}\right), \mathrm{Pb}\left(27.23 \mu \mathrm{g} . \mathrm{g}^{-1}\right)$, $\mathrm{Cd}\left(10.497 \mu \mathrm{g} \cdot \mathrm{g}^{-1}\right)$, Syntrichia ruralis for Fe (1419.02 $\left.\mu \mathrm{g} . \mathrm{g}^{-1}\right)$, Mn $\left(246.70 \mu \mathrm{g} \cdot \mathrm{g}^{-1}\right), \mathrm{Zn}\left(35.28 \mu \mathrm{g} . \mathrm{g}^{-1}\right)$, Ni $\left(86.74 \mu \mathrm{g}^{\mathrm{g}-1}\right), \mathrm{Cr}\left(50.71 \mu \mathrm{g} . \mathrm{g}^{-1}\right), \mathrm{Cu}\left(25.17 \mu \mathrm{g} . \mathrm{g}^{-1}\right), \mathrm{Pb}$ $\left(30.38 \mu \mathrm{g} \cdot \mathrm{g}^{-1}\right), \mathrm{Cd}\left(22.684 \mu \mathrm{g} \cdot \mathrm{g}^{-1}\right)$ and Bryum capillare for $\mathrm{Fe}\left(608.36 \mu \mathrm{g} . \mathrm{g}^{-1}\right), \mathrm{Mn}\left(30.01 \mu \mathrm{g} . \mathrm{g}^{-1}\right), \mathrm{Zn}(11.89$ $\left.\mu \mathrm{g} . \mathrm{g}^{-1}\right), \mathrm{Ni}\left(34.39 \mu \mathrm{g} \cdot \mathrm{g}^{-1}\right), \mathrm{Cr}\left(31.68 \mu \mathrm{g} \cdot \mathrm{g}^{-1}\right), \mathrm{Cu}(11.72$ $\left.\mu \mathrm{g} \cdot \mathrm{g}^{-1}\right), \quad \mathrm{Pb} \quad\left(17.71 \mu \mathrm{g} . \mathrm{g}^{-1}\right), \quad \mathrm{Cd}\left(5.627 \mu \mathrm{g} \cdot \mathrm{g}^{-1}\right)$ respectively.

Iron is the most abundant element in the biosphere. Primary iron sources are coming from mining, erosion and intense traffic (Rühling and Steinnes, 1998; Namik et al., 2006). The highest and lowest iron amount were determined as $1535.4 \mu \mathrm{g} \cdot \mathrm{g}^{-1}$ in Homalothecium

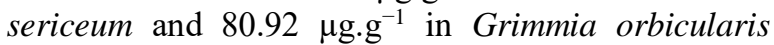
respectively (Figure 2; Table 2). This data is higher than the earliest reported studies (Harmens et al., 2010; Ruhling and Taylor, 2004; Herpin et al., 1997; Vargha et al., 2002; Adamo et al., 2003; Giordano et al., 2005; Barandovski et al., 2008; Uyar et al., 2008).

Table 2. Level of heavy metals in moss species $\left(\mu \mathrm{g} \cdot \mathrm{g}^{-1}\right)$.

\begin{tabular}{|c|c|c|c|c|c|c|c|c|c|}
\hline Moss species & $\begin{array}{l}\text { Area } \\
\text { (SN) }\end{array}$ & $\mathbf{C u}$ & $\mathbf{P b}$ & Cd & Zn & $\mathbf{F e}$ & $\mathrm{Cr}$ & $\mathbf{N i}$ & Mn \\
\hline \multirow{4}{*}{ Homalothecium sericeum } & U (1) & $5.95 \pm 6.0$ & $8.16 \pm 0.8$ & $5.228 \pm 0.5$ & BDL & $178.38 \pm 17.8$ & $12.41 \pm 1.2$ & $15.9 \pm 1.6$ & $\mathrm{BDL}$ \\
\hline & $\mathbf{P}(2)$ & $8.27 \pm 0.8$ & $10.2 \pm 1.0$ & $7.627 \pm 0.8$ & $19.7 \pm 1.9$ & $544.57 \pm 54.46$ & $12.37 \pm 1.2$ & $21.3 \pm 2.1$ & BDL \\
\hline & $\mathbf{P}(3)$ & $24.02 \pm 2.4$ & $11.35 \pm 1.1$ & $6.623 \pm 07$ & $158.44 \pm 15.8$ & $932.38 \pm 93.2$ & $22.05 \pm 2.2$ & $24.51 \pm 2.5$ & $233.6 \pm 23.4$ \\
\hline & $\mathbf{P}(4)$ & $16.25 \pm 1.6$ & $8.19 \pm 0.8$ & $9.185 \pm 0.9$ & $26.39 \pm 2.64$ & $1535.4 \pm 153.5$ & $60.51 \pm 6.1$ & $87 \pm 8.7$ & $186.18 \pm 18.6$ \\
\hline \multirow{2}{*}{ Bryum capillare } & $\mathrm{U}(5)$ & $3.81 \pm 0.4$ & BDL & $5.627 \pm 0.5$ & BDL & $238.98 \pm 23.9$ & $6.04 \pm 0.6$ & $3.15 \pm 0.3$ & BDL \\
\hline & $\mathbf{P}(6)$ & $11.72 \pm 1.2$ & $17.71 \pm 1.8$ & $6.647 \pm 0.7$ & $11.89 \pm 1.2$ & $608.36 \pm 60.8$ & $31.68 \pm 3.2$ & $34.39 \pm 3.4$ & $30.01 \pm 3.0$ \\
\hline \multirow{11}{*}{ Syntrichia ruralis } & $\mathrm{U}(7)$ & $4.84 \pm 0.5$ & $1.83 \pm 0.2$ & $5.924 \pm 0.6$ & $1.984 \pm 0.2$ & $140.60 \pm 14.0$ & $9.20 \pm 0.9$ & $19.49 \pm 1.9$ & BDL \\
\hline & $\mathbf{P}(\mathbf{8})$ & $10.69 \pm 1.1$ & $11.33 \pm 1.1$ & $6.630 \pm 0.7$ & $2.22 \pm 0.2$ & $1267.98 \pm 126.8$ & $44.36 \pm 4.4$ & $86.74 \pm 8.7$ & $165.32 \pm 16.5$ \\
\hline & $\mathbf{P}(\mathbf{9})$ & $14.66 \pm 1.5$ & $23.98 \pm 2.4$ & $10.543 \pm 1.1$ & $34.35 \pm 3.4$ & $463.86 \pm 46.4$ & $25.19 \pm 2.5$ & $32.65 \pm 3.3$ & $23.15 \pm 2.3$ \\
\hline & $\mathbf{P}(\mathbf{1 0})$ & $10.42 \pm 1.0$ & $14.52 \pm 1.5$ & $12.253 \pm 1.2$ & $24.76 \pm 2.5$ & $344.43 \pm 34.4$ & $25.25 \pm 2.5$ & $32.72 \pm 3.3$ & $36.77 \pm 3.7$ \\
\hline & $\mathbf{P}(11)$ & $11.15 \pm 1.1$ & $23.97 \pm 2.4$ & $9.949 \pm 1.0$ & $18.27 \pm 1.8$ & $816.92 \pm 81.7$ & $44.33 \pm 4.4$ & $58.84 \pm 5.9$ & $158.45 \pm 15.8$ \\
\hline & $\mathbf{P}(12)$ & $11.83 \pm 1.2$ & $11.35 \pm 1.1$ & $8.935 \pm 0.9$ & $19.93 \pm 2.0$ & $1007.02 \pm 100.7$ & $25.24 \pm 2.5$ & $45.85 \pm 4.6$ & $111.37 \pm 11.1$ \\
\hline & $\mathbf{P}(13)$ & $18.68 \pm 1.9$ & $30.38 \pm 3.0$ & $10.628 \pm 1.1$ & $36.04 \pm 3.6$ & $1120.40 \pm 112.0$ & $34.86 \pm 3.5$ & $52.44 \pm 5.2$ & $152.13 \pm 15.2$ \\
\hline & $\mathbf{P}(14)$ & $7.99 \pm 0.8$ & $5.8 \pm 0.5$ & $8.942 \pm 0.9$ & $3.83 \pm 0.4$ & $502.74 \pm 50.3$ & $18.85 \pm 1.9$ & $32.74 \pm 3.3$ & $16.43 \pm 1.6$ \\
\hline & $\mathbf{P}(15)$ & $22.83 \pm 2.3$ & $14.50 \pm 1.5$ & $22.684 \pm 2.3$ & $66.52 \pm 6.7$ & $1419.02 \pm 141.9$ & $44.38 \pm 4.4$ & $55.63 \pm 5.6$ & $246.70 \pm 24.7$ \\
\hline & P (16) & $25.17 \pm 2.5$ & $11.33 \pm 1.1$ & $9.098 \pm 0.9$ & $35.28 \pm 3.5$ & $667.55 \pm 66.8$ & $44.38 \pm 4.4$ & $42.50 \pm 4.3$ & $138.31 \pm 13.8$ \\
\hline & $\mathbf{P}(\mathbf{1 7})$ & $13.42 \pm 1.3$ & $5.00 \pm 0.5$ & $9.939 \pm 1.0$ & $23.09 \pm 2.3$ & $1342.27 \pm 134.2$ & $50.71 \pm 5.1$ & $53.92 \pm 5.4$ & $185.52 \pm 18.6$ \\
\hline \multirow{4}{*}{ Grimmia orbicularis } & U (18) & $6.55 \pm 0.7$ & $5.00 \pm 0.5$ & $7.198 \pm 0.7$ & BDL & $80.92 \pm 8.1$ & $2.84 \pm 0.3$ & $3.15 \pm 0.3$ & $29.94 \pm 3.0$ \\
\hline & $\mathbf{P}(19)$ & $20.64 \pm 2.1$ & $27.23 \pm 2.7$ & $10.497 \pm 1.0$ & $40.90 \pm 4.1$ & $585.88 \pm 58.6$ & $25.27 \pm 2.5$ & $37.69 \pm 3.8$ & $125.10 \pm 12.5$ \\
\hline & $\mathbf{P}(20)$ & $17.17 \pm 1.7$ & $23.99 \pm 2.4$ & $7.997 \pm 0.8$ & $42.40 \pm 4.2$ & $599.39 \pm 59.9$ & $18.82 \pm 1.9$ & $37.59 \pm 3.8$ & $32.17 \pm 3.2$ \\
\hline & $\mathbf{P}(21)$ & $17.59 \pm 1.8$ & $27.20 \pm 2.7$ & $9.687 \pm 1.0$ & $52.13 \pm 5.2$ & $856.82 \pm 85.7$ & $34.85 \pm 3.5$ & $57.36 \pm 5.7$ & $213.17 \pm 21.3$ \\
\hline \multirow{8}{*}{ Homalothecium lutescens } & U (22) & $5.90 \pm 0.6$ & $8.2 \pm 0.8$ & $5.23 \pm 0.5$ & BDL & $180.32 \pm 18.0$ & $12.41 \pm 1.2$ & $34.60 \pm 3.5$ & BDL \\
\hline & $\mathbf{P}(23)$ & $17.21 \pm 1.7$ & $23.95 \pm 2.4$ & $9.643 \pm 1.0$ & $69.59 \pm 7.0$ & $500.71 \pm 50.0$ & $31.54 \pm 3.2$ & $37.51 \pm 3.8$ & $104.24 \pm 10.4$ \\
\hline & $\mathbf{P}(24)$ & $13.68 \pm 1.4$ & $27.22 \pm 2.7$ & $12.014 \pm 1.2$ & $18.34 \pm 1.8$ & $615.89 \pm 61.6$ & $31.68 \pm 3.2$ & $37.10 \pm 3.7$ & $43.59 \pm 4.4$ \\
\hline & $\mathbf{P}(25)$ & $15.17 \pm 1.5$ & $24.02 \pm 2.4$ & $13.296 \pm 1.3$ & $2.22 \pm 0.2$ & $1270.15 \pm 127.0$ & $47.63 \pm 4.8$ & $78.68 \pm 7.9$ & $152.04 \pm 15.2$ \\
\hline & $\mathbf{P}(\mathbf{2 6})$ & $11.14 \pm 1.1$ & $27.20 \pm 2.7$ & $19.262 \pm 1.9$ & $39.25 \pm 3.9$ & $1195.30 \pm 119.5$ & $31.64 \pm 3.2$ & $45.85 \pm 4.6$ & $97.82 \pm 9.8$ \\
\hline & $\mathbf{P}(27)$ & $14.33 \pm 1.4$ & $24.04 \pm 2.4$ & $9.411 \pm 0.9$ & $15.11 \pm 1.5$ & $487.60 \pm 48.8$ & $25.26 \pm 2.5$ & $42.59 \pm 4.3$ & $125.01 \pm 12.5$ \\
\hline & $\mathbf{P}(28)$ & $13.75 \pm 1.4$ & $18.3 \pm 1.8$ & $8.926 \pm 0.9$ & $34.30 \pm 3.4$ & $853.77 \pm 85.4$ & $50.67 \pm 5.1$ & $109.53 \pm 11.0$ & $219.17 \pm 21.9$ \\
\hline & $\mathbf{P}(29)$ & $18.73 \pm 1.9$ & $23.7 \pm 2.3$ & $35.866 \pm 3.6$ & $34.45 \pm 3.4$ & $1271.93 \pm 127.2$ & $60.51 \pm 6.1$ & $52.48 \pm 5.2$ & $362.77 \pm 36.3$ \\
\hline
\end{tabular}

(U: Unpolluted; P: Polluted; SN: Sample Number) 


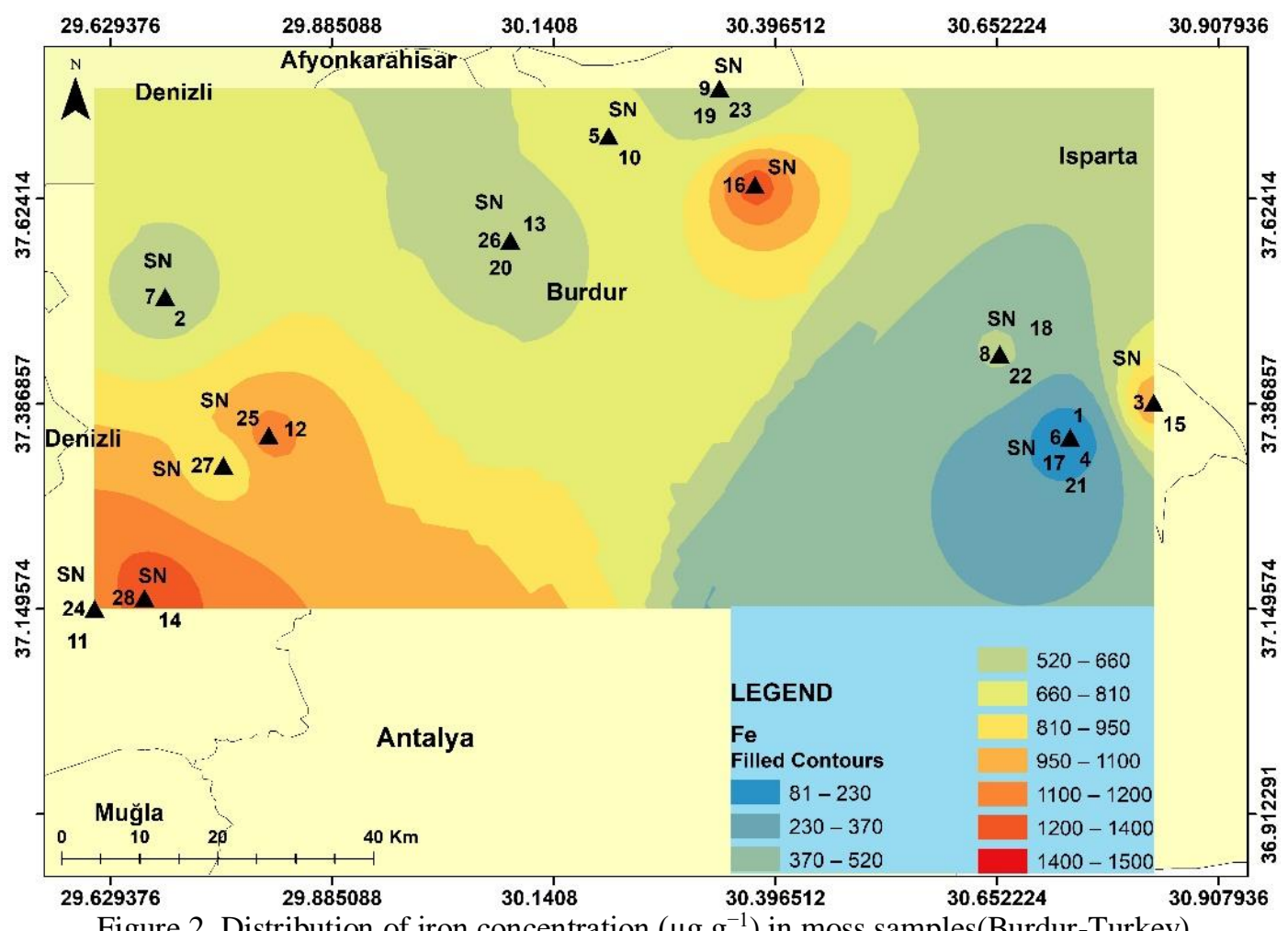

Figure 2. Distribution of iron concentration $\left(\mu \mathrm{g} \cdot \mathrm{g}^{-1}\right)$ in moss samples(Burdur-Turkey)

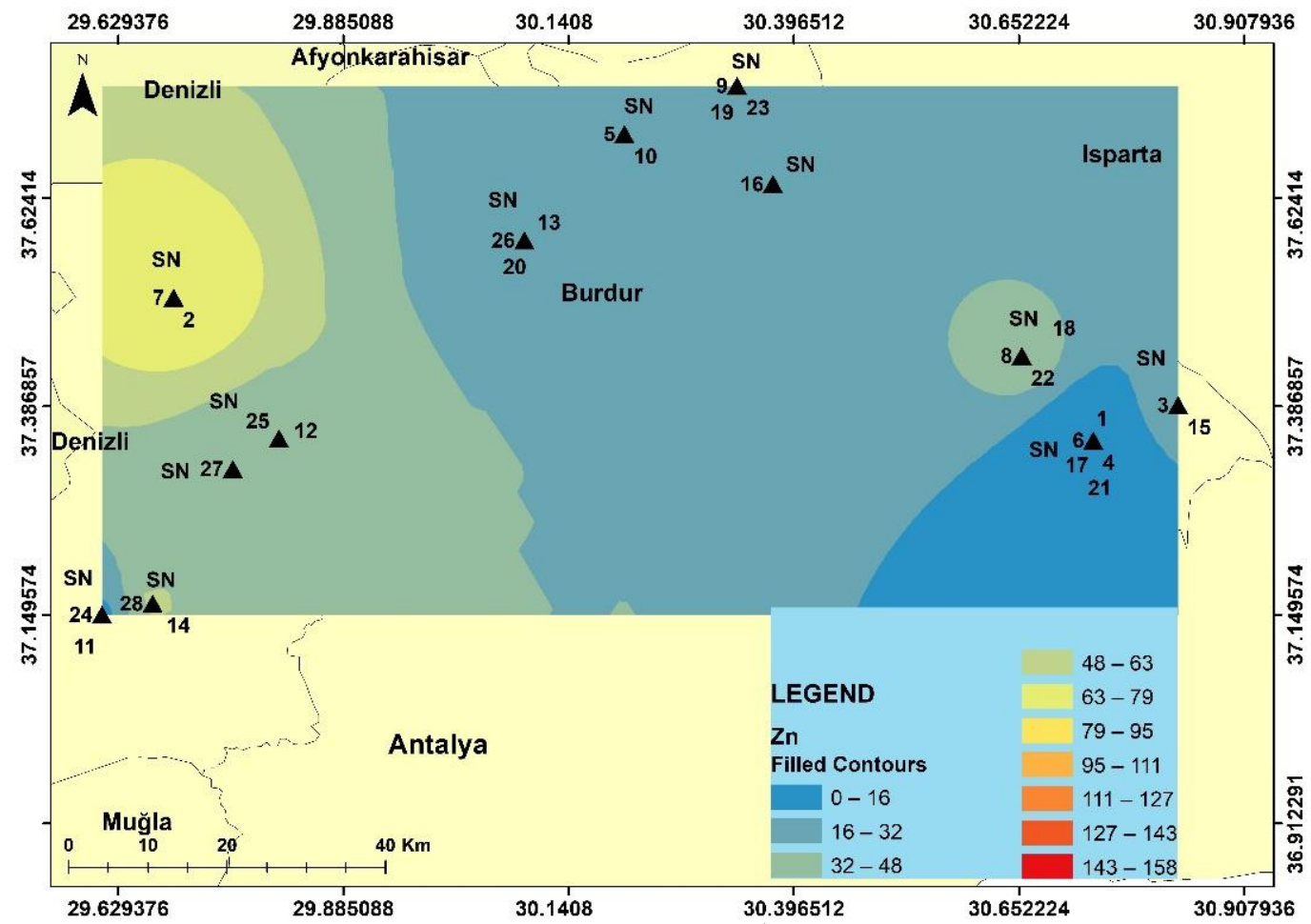

Figure 3. Distribution of zinc concentration ( $\mu \mathrm{g} . \mathrm{g}^{-1}$ ) in moss samples(Burdur-Turkey)

Zinc is a necessary element for tissues. For this reason many bio molecules for example proteins, carbohydrates, lipids, DNA, RNA and ATP involve zinc (Strain and Cashman, 2009). Zn concentrations were ranged from $1.984 \mu \mathrm{g} .{ }^{\mathrm{g}-1}$ to $158.44 \mu \mathrm{g} . \mathrm{g}^{-1}$ in Syntrichia ruralis (Figure 3, Table 2). Zinc values are parallel to the literature 14-203 $\mu \mathrm{g} . \mathrm{g}^{-1}$ (Barandovski et al., 2008). Zinc amount was detected very high level according to literature in Syntrichia ruralis with 235.28 $\mu \mathrm{g} \cdot \mathrm{g}^{-1}$. This amount can be toxic for whole organisms in the near vicinity.

Chromium levels were ranked 2.84-60.52 $\mu \mathrm{g} . \mathrm{g}^{-1}$ in moss samples (Figure 4; Table 2). Chromium values are 
higher than the literature values which were detected for many European countries (Harmens et al., 2010; Ruhling and Taylor, 2004; Vargha et al., 2002; Giordano et al., 2005; Barandovski et al., 2008; Uyar et al., 2008). Basic chromium sources are coming from intensive traffic, coal-fired power plants and coalmining (Uyar et al., 2007a;b).

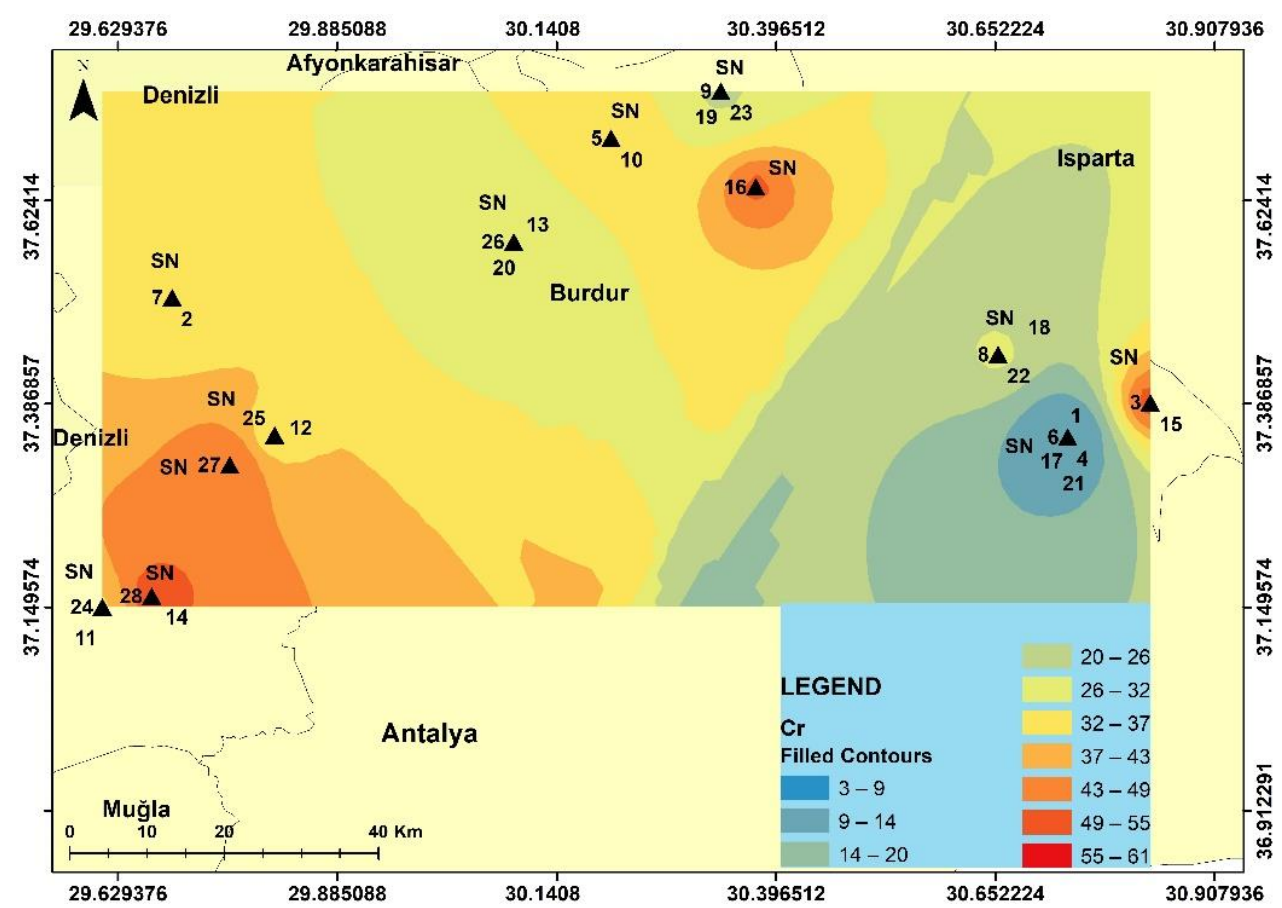

Figure 4. Distribution of chromium concentration $\left(\mu \mathrm{g} . \mathrm{g}^{-1}\right)$ in moss samples(Burdur-Turkey)

Copper concentration was found $4.84-25.17 \mu \mathrm{g} \cdot \mathrm{g}^{-1}$ in the moss samples (Figure 5; Table 2). This results were higher than Ruhling and Taylor (2004); Vargha et al. (2002) and Uyar et al. (2009), but Giordona et al. (2005) which were reported higher values than our results (Giordano et al., 2005). Copper mostly comes from mining, traffic, fungicides and fertilizers used by agriculture and in fact from soil (Rühling and Steinnes, 1998).

Copper is widely used as manure and nutritional supplements and due to its antimicrobial and antifungal properties, as fungicides and insecticides in agriculture (Strain and Cashman, 2009).

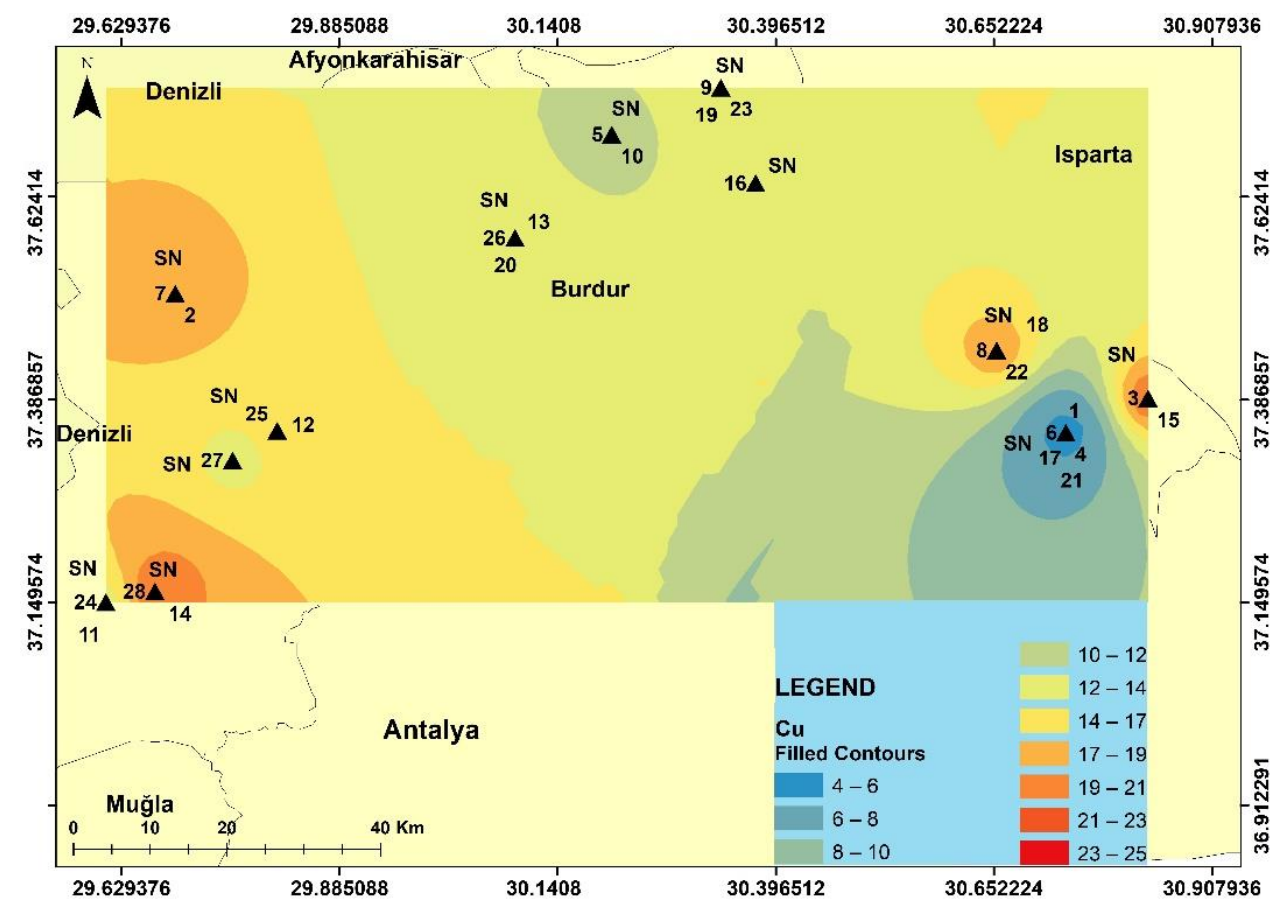

Figure 5. Distribution of copper concentration $\left(\mu \mathrm{g} \cdot \mathrm{g}^{-1}\right)$ in moss samples(Burdur-Turkey) 


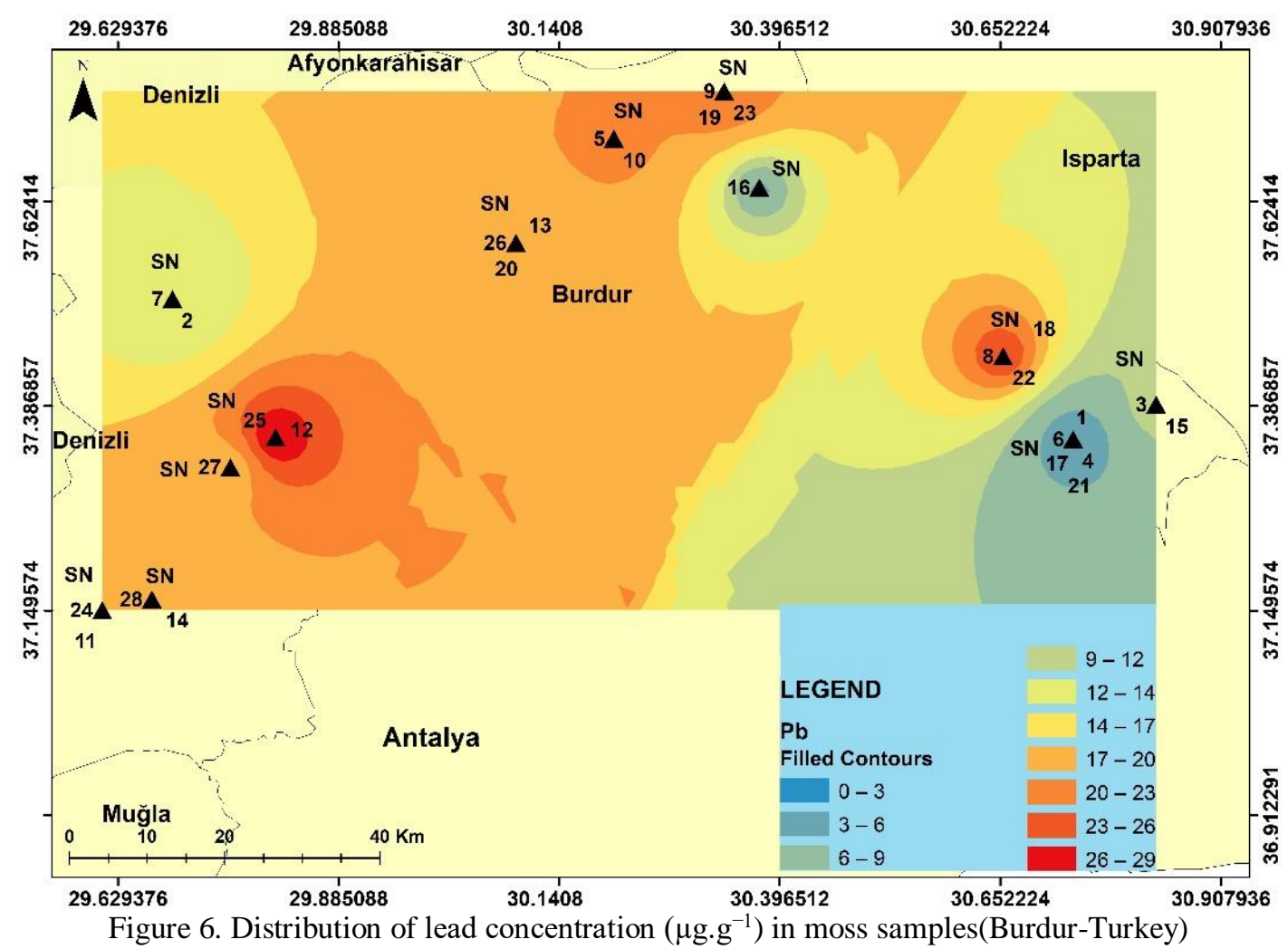

The lead level was found as 8.16-20.38 $\mu \mathrm{g} \cdot \mathrm{g}^{-1}$ in the moss samples (Table 2; Figure 6). The accumulation of lead to in the petrol increases the concentration of lead. In literature, the high lead concentration reported in the samples collected from high-density traffic areas (Tüzen et al., 2003). Lead amount is lower than according to Harmens et al. (2010), Vargha et al.

(2002), Giordano et al. (2005), Barandovski et al. (2008), Uyar et al. (2008), Ares et al. (2011). Combustion of fossil fuels (including lead) are still the main reason for lead pollution, other lead sources like vehicles, coal combustion and mining (Uyar et al., 2008).

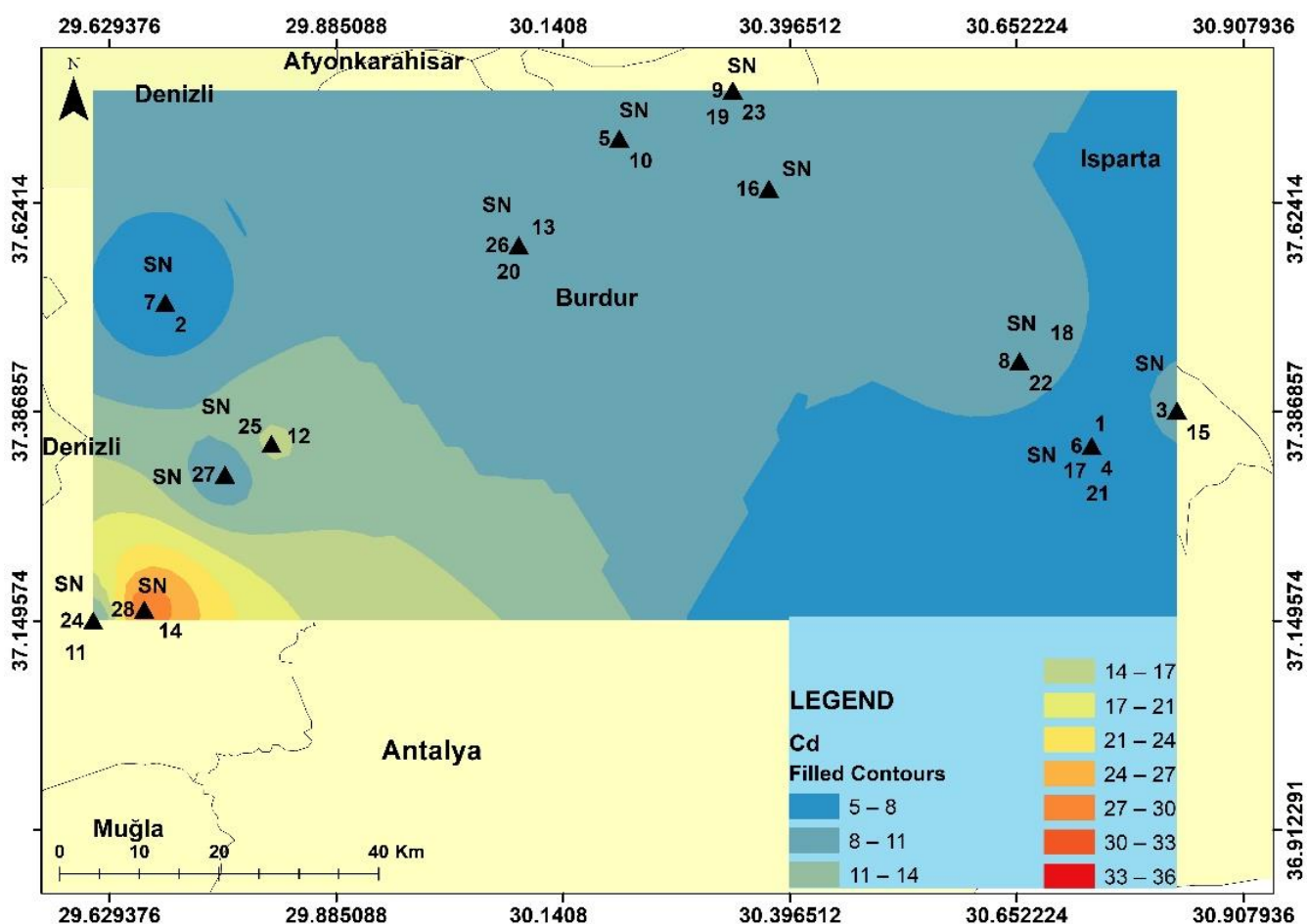

Figure 7. Distribution of cadmium concentration $\left(\mu \mathrm{g} \cdot \mathrm{g}^{-1}\right)$ in moss samples(Burdur-Turkey) 
The increase in the level of Cadmium influences numerous metabolic activities such as photosynthesis, respiration, ion uptake, growth and development, with the effect on the organisms, soil fertility and ecosystem activities. Cadmium concentration was found as 5.2-35.8 $\mu \mathrm{g} . \mathrm{g}^{-1}$ in the moss samples (Figure 7; Table 2). These values were higher than reported in the literature.

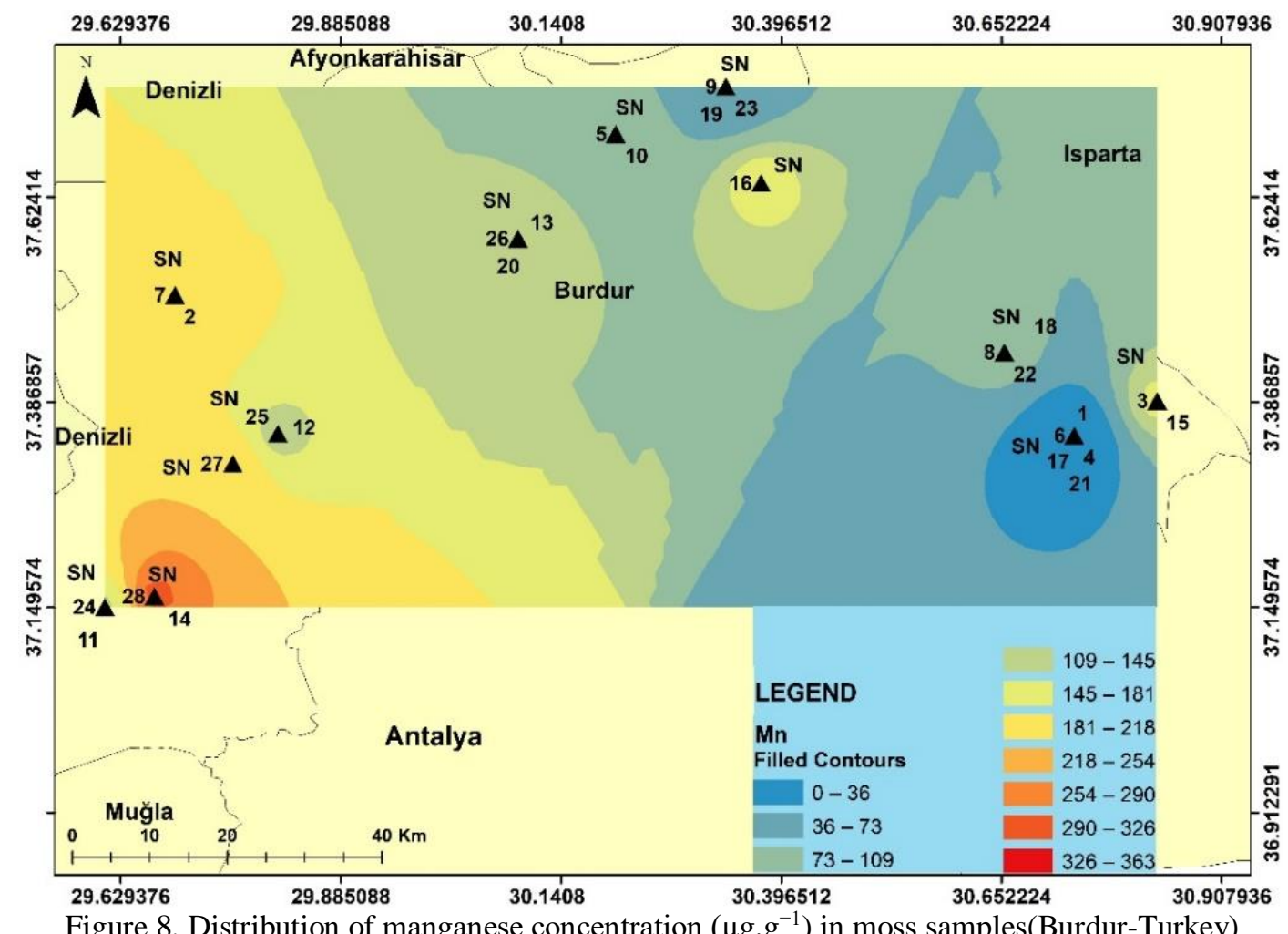

The manganese concentration was $16.43-362.7 \mu \mathrm{g} \cdot \mathrm{g}^{-1}$ in the mosses samples (Figure 8; Table 2). These values are in agreement with reported data from the literature (Sari et al., 2005; Özdemir and Mendil, 2008; Özdemir et al., 2010). Toxicity limits of manganese for plants are high (400-1000 $\left.\mu \mathrm{g} \cdot \mathrm{g}^{-1}\right)$. Present manganese level is lower than the literature.

Nickel can bind strongly to molecules such as ribonucleic acid in the organism. Amino acids such as cysteine, methionine and histidine can be combined with components such as phospholipids, acetyl CoA and citric acid.

Nickel mainly originates from oil and coal burning, the steel industry, and smelters (Ötvös et al., 2003). Average nickel levels in the whole study area were approximately in the range of 3.15 and $109.5 \mu \mathrm{g} . \mathrm{g}^{-1}$ (Figure 9; Table 2). These nickel values were quite high compared to the European countries. 


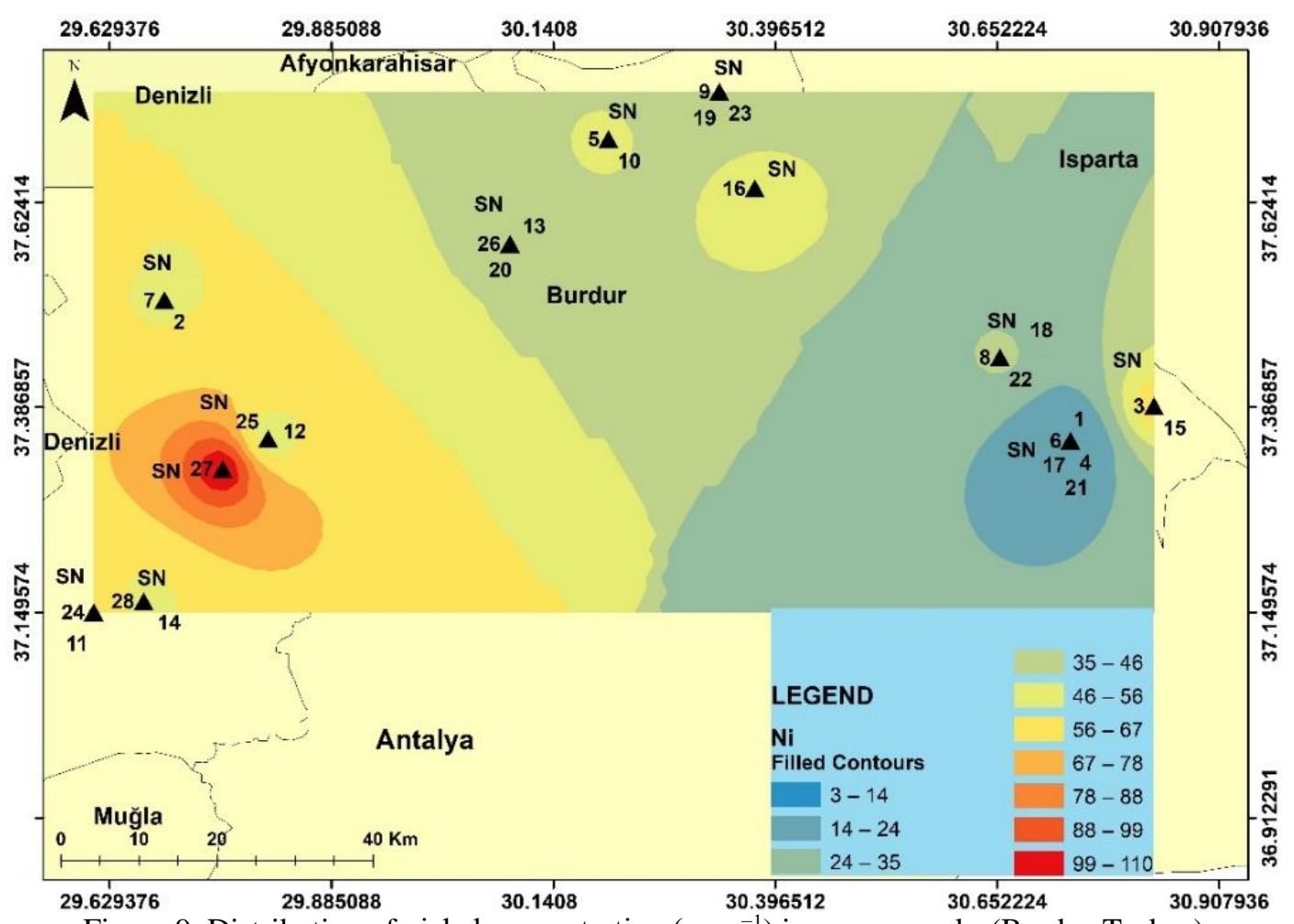

Figure 9. Distribution of nickel concentration ( $\mu \mathrm{g} . \mathrm{g}^{-1}$ ) in moss samples(Burdur-Turkey)

We can say that the mosses are one of the best bioindicators that can be used to determine heavy metal pollution and the element levels in the mosses collected from the highway sides in the study area were showed differences depending on the moss species and the condition of the location of the mosses.

\section{Conclusion}

Increasing industrial activities, number of vehicles and human population in recent years, excessive and unconscious chemical fertilizers and pesticide applications, mixing of wastewater into water resources and use of irrigation water increase the content of heavy metals in soil, water, atmosphere and plants. The increase in heavy metals in soil, water, atmosphere and plants affects many metabolic activities such as photosynthesis, respiration, ion uptake, growth and development, with its effect on plant life, soil fertility and ecosystem activities. It affects these metabolic activities, leading to a decrease in yield and quality. In addition to reaching human and animal food chains through plants, heavy metals are taken by the body through respiration lead to diseases such as constant headaches, dizziness, nausea, vomiting, insomnia, asthma, and bone erosion. Also, heavy metals and their components accumulate in the tissue of bone, muscle, blood, kidneys and liver. They can cause major diseases such as high blood pressure, lung cancer, bone erosion, anemia and so on.

These results show that there is pollution on the close to agricultural areas and roads with intense vehicle traffic such as Isparta-Antalya, Burdur-Denizli,
Burdur-Afyonkarahisar, Burdur-Muğla (Fethiye) and Burdur-Antalya highways.

\section{References}

Adamo P. Giordano S. Vingiani S. Cobianchi R.C. Violente P. 2003. Trace element accumulation by moss and lichen exposed in bags in the city of Naples (Italy). Environmental Pollution. 122, 91-103.

Akçay N. Batan N. Çınar Y. 2016. Investigate of atmospheric arsenic, cadmium, chromium, lead, and mercury levels in moss species found around Zilkale, by EDXRF Spectrometry. AIP Conference Proceedings, 1726, 020124. https://doi.org/10.1063/1.4945950.

Ares A. Fernandez J.A. Aboal J.R. Carballeira A. 2011. Study of the air quality in industrial areas of Santa Cruz de Tenerife (Spain) by active biomonitoring with Pseudoscleropodium purum. Ecotoxicology and Environmental Safety. 74, 533-541.

Barandovski L. Cekova M. Frontasyeva M.V. Pavlov S.S. Stafilov T. Steinnes E. Urumov V. 2008. Atmospheric deposition of trace element pollutions in Macedonia studied by the moss biomonitoring technique. Environmental Monitoring and Assessment. 138, 107-118.

Brown D.H. Bates J.W. 1990. Bryophytes and nutrient cycling. Botanical Journal of the Linnean Society. 104, 129-147.

Chakrabortty S. Paratkar G.T. 2006. Biomonitoring of trace element air pollution using mosses. Aerosol and Air Quality Research. 6:3, 247-258. 
Farid G. Sarwar N. Ahmad A. Ghafoor A. Rehman M. 2015. Heavy Metals (Cd, $\mathrm{Ni}$ and $\mathrm{Pb})$ Contamination of Soils, Plants and Waters in Madina Town of Faisalabad Metropolitan and Preparation of Gis Based Maps. Advances in Crop Science and Technology. 4: 199. doi:10.4172/2329-8863.1000199.

Giordano S. Adamo P. Sorbo S. Vingiana S. 2005. Atmospheric trace metal pollution in the Naples urban area based on results from moss and lichen bags. Environmental Pollution. 136, 431442.

Harmens H. Norris D.A. Steinnes E. Kubin E. Piispanen J. Alber R. Aleksiayenak Y. Blum O. Coşkun M. Dam M. et al. 2010. Mosses as biomonitors of atmospheric heavy metal deposition: Spatial patterns and temporal trends in Europe. Environmental Pollution. 158, 31443156.

Herpin U. Markert B. Weckert V. Berlecamp J. Friese K. Siewers U. Lieth H. 1997. Retrospective analysis of heavy metal concentrations at selected locations in the Federal Republic of Germany using moss material from a herbarium. Science of the Total Environment. 205, 1-12.

Namik K. Aras O. Ataman Y. 2006. Trace Element Analiysis of Food and Diet (Chapter 1: Importance of trace elements in Food). Published by the Royal Society of Chemistry, Cambridge.

Onianwa P.C. 2001. Monitoring athmospheric metal pollution: A review of the use of mosses as indicators.

Environmental Monitoring and Assessment. 71, 13-50.

Ötvös E. Pazmandi T. Tuba Z. 2003. First national survey of atmospheric heavy metal deposition in Hungary by the analysis of mosses. Science of the Total Environment. 309, 151-160.

Özdemir T. Apaydin G. Mendil D. Bulut V.N. Cengiz E. Gündoğdu A. Aylikci V. 2010. Determination of some elements in moss samples from North Eastern Anatolia, Turkey. Asian Journal of Chemestry. 22, 1, 346-352.

Özdemir T. Mendil D. 2008. Determination of trace elements in some moss samples by atomic absorption spectrometry. Asian Journal of Chemestry. 20:3, 1786-1790.

Rühling A. Steinnes E. 1998. Atmospheric heavy metal deposition in Europe 1995-1996. Nordic Council of Ministers (ed) 15, 1-67.
Ruhling A. Taylor G. 2004. Changes in the atmospheric deposition of minor and rare elements between 1975-2000 in South Sweden, as measured by moss analysis. Environmental Pollution. 131, 417- 423.

Sari H. Mendil D. Tüzen M. Hasdemir E. Özdemir T. 2005. AAS Determination Of Trace Metals in Some Moss Samples From Trabzon Turkey. Fresenius Environmental Bulletin. 14:6, 473477.

Strain J.J. Cashman K.D. 2009. Minerals and trace elements (in: Introduction to human nutrition, second edition).

Tüzen M. Mendil D. Sarı H. Hasdemir E. 2003. AAS Determination of Heavy Metals in Moss Samples of Giresun-Turkey. Fresenius Environmental Bulletin. 12:10, 1283-1286.

Tyler G. 1990. Bryophytes and heavy metals: a literature review. Botanical Journal of the Linnean Society. 104, 231-253.

Uyar G. Ören M. Yildirim Y. Ince M. 2007a. Mosses as indicators of atmospheric heavy metal deposition around a coal-fired power plant in Turkey. Fresenius Environmental Bulletin. 16:2, 182-192.

Uyar G. Ören M. Ince M. 2007b. Atmospheric heavy metal deposition in Düzce province by using mosses as biomonitors. Fresenius Environmental Bulletin. 16:2, 145-153.

Uyar G. Ören M. Yildirim Y. Öncel S. 2008. Biomonitoring of metal deposition in the vicinity of Eregli steel plant in Turkey. Environmental Forensics. 9, 350-363.

Uyar G. Avcil E. Ören M. Karaca F. Oncel M.S. 2009. Determination of heavy metal pollution in Zonguldak (Turkey) by moss analysis (Hypnum cupressiforme). Environmental Engineering Science. 26:1, 183-194.

Vargha B. Ötvös E. Tuba Z. 2002. Investigations on ecological effects of heavy metal pollution in Hungary by mossdwelling water bears (Tardigrada), as bioindicators. Annals of agricultural and environmental medicine 9: 141-146.

Zeichmeister H.G. Grodzinska K. Szarek-Lukaszewska G. 2003. Bryophytes. Markert, BA. Breure, A.M. \& Zeichmeister, H. G.(Eds.). Elsevier, Oxford. 